\title{
Polymeric nanoparticle-based delivery of microRNA-199a-3p inhibits proliferation and growth of osteosarcoma cells
}

This article was published in the following Dove Press journal:

International Journal of Nanomedicine

I5 April 2015

Number of times this article has been viewed

\author{
Linlin Zhang ${ }^{1,2, *}$ \\ Arun K lyer ${ }^{3,4, *}$ \\ Xiaoqian Yang' \\ Eisuke Kobayashi' \\ Yuqi Guo',2 \\ Henry Mankin' \\ Francis J Hornicek' \\ Mansoor M Amiji ${ }^{3}$ \\ Zhenfeng Duan'
}

'Sarcoma Biology Laboratory, Center for Sarcoma and Connective Tissue Oncology, Massachusetts General

Hospital, Boston, Massachusetts, USA; ${ }^{2}$ Department of Pathology, The Third

Affiliated Hospital of Zhengzhou

University, Zhengzhou, People's

Republic of China; ${ }^{3}$ Department of Pharmaceutical Sciences.

School of Pharmacy, Northeastern University, Boston, Massachusetts, USA; ${ }^{4}$ Department of Pharmaceutical Sciences, Eugene Applebaum College of Pharmacy and Health Sciences, Wayne State University, Detroit, MI, USA

*These authors contributed equally to this work

Correspondence: Zhenfeng Duan Sarcoma Biology Laboratory, Center for Sarcoma and Connective Tissue Oncology, Massachusetts General Hospital, 100 Blossom St, Jackson III5, Boston, MA 02II4, USA

$\mathrm{Tel}+\mathrm{I} 6177243144$

Fax +I 6177263883

Email zduan@mgh.harvard.edu
Abstract: Our prior screening of microRNAs (miRs) identified that miR-199a-3p expression is reduced in osteosarcoma cells, one of the most common types of bone tumor. miR-199a-3p exhibited functions of tumor cell growth inhibition, suggesting the potential application of miR-199a-3p as an anticancer agent. In the study reported here, we designed and developed a lipid-modified dextran-based polymeric nanoparticle platform for encapsulation of miRs, and determined the efficiency and efficacy of delivering miR-199a-3p into osteosarcoma cells. In addition, another potent miR, let-7a, which also displayed tumor suppressive ability, was selected as a candidate miR for evaluation. Fluorescence microscopy studies and real-time polymerase chain reaction results showed that dextran nanoparticles could deliver both miR199a-3p and let-7a into osteosarcoma cell lines (KHOS and U-2OS) successfully. Western blotting analysis and 3-(4,5-dimethylthiazol-2-yl)-2,5-diphenyltetrazolium bromide (MTT) assays demonstrated that dextran nanoparticles loaded with miRs could efficiently downregulate the expression of target proteins and effectively inhibit the growth and proliferation of osteosarcoma cells. These results demonstrate that a lipid-modified dextran-based polymeric nanoparticle platform may be an effective nonviral carrier for potential miR-based anticancer therapeutics.

Keywords: bone tumor, dextran nanoparticles, miR-199a-3p, let-7a, RNAi

\section{Introduction}

Osteosarcoma is the most common primary malignant tumor of bone. It usually occurs in the developing bones of children and adolescents. ${ }^{1}$ Currently, the treatment of osteosarcoma involves surgery, radiation therapy, and adjuvant chemotherapy. ${ }^{2}$ Despite recent advances in chemotherapy, the 5-year event-free survival and overall survival rates are still around $60 \% .{ }^{2,3}$ Furthermore, drug resistance to chemotherapy frequently develops in osteosarcoma and dose-limiting toxicity restricts the utility of chemotherapeutic drugs. Thus, more selective and effective therapeutic strategies are required for the treatment of osteosarcoma. ${ }^{3-6}$

Recent years have seen remarkable progress made in basic understanding of the disease and in deciphering the role of microRNAs (miRs) in cancer. "miRs" are a subset of small noncoding RNA molecules that influence tumor formation, maintenance, metastasis, apoptosis, and drug resistance. Mature miRs bind to the $3^{\prime}$ untranslated regions of target genes and inhibit gene expression by degradation or repress translation of the target messenger RNA. There has been great interest in the function of miRs in human cancers and numerous studies have observed the dysregulation of miRs in different tumors, including osteosarcoma. ${ }^{7-12}$ In this regard, we recently demonstrated that the expression of miR-199a-3p is remarkably decreased in osteosarcoma cell lines. 
The transfection of miR-199a-3p into osteosarcoma cells can significantly decrease cell growth and proliferation. ${ }^{11}$ The expression of miR-199a-3p is also downregulated in several human malignancies including colon and hepatocellular carcinoma. ${ }^{13-15}$ Restoring the expression of miR-199a-3p in these tumor cells led to a reduced invasive capability of cancer cells and increased sensitivity to chemotherapeutic drugs. ${ }^{13}$ These results suggest that miR-199a-3p can be used as a potential treatment target for such cancers. Similarly, another tumor suppressor miR, let-7a, has been found downregulated or completely repressed in many types of human cancers. ${ }^{16-19}$ Accordingly, the restoration of let-7a expression has been found to inhibit the proliferation of many cancer cells. ${ }^{17,20-23}$ Thus, the exogenous transfection of specific miR into tumor cells may open up newer avenues for the effective treatment of several human cancers.

Although miR-based anticancer strategies are emerging as a highly promising therapeutic approach, their systemic delivery still remains a great challenge. Similar to small interfering RNA (siRNA) molecules, miRs are highly unstable in the cell environment and must be delivered by effective carrier vectors. ${ }^{24}$ While viral vectors may also be used for proof-of-concept experimental approaches to the cellular delivery of miRs, safe and efficient nonviral delivery systems are required in order to translate their utility into clinically viable therapeutic strategies that can benefit cancer patients. In this regard, polymeric nanoparticle-based delivery serves as a promising platform with several advantages, including higher transfection efficiencies, targeted delivery, and ease of modification or functionalization, and has the added benefit of safety and nontoxicity. ${ }^{25}$ Several reports have shown that miRs can be delivered into the cancer cell by nano-sized nonviral vectors. For example, liposome-polycation-hyaluronic acid modified with a GC4 single-chain variable antibody $(\mathrm{scFv})$ was used to deliver miR-34a into B16F10 lung cancer cells. ${ }^{26}$ Cysteamine-functionalized gold nanoparticles were able to deliver miRs into two different tumor models, and the results showed that a $96 \%$ transfection rate of miRs into cells was achieved and $98 \%$ of cells showed good viability following treatment. The data also showed these gold nanoparticles had a ten- to 20 -fold higher payload capacity than Lipofectamine ${ }^{\circledR} .{ }^{27}$ Several novel nanotechnology-based $\mathrm{miR} /$ siRNA delivery platforms are still in the early stages of development. ${ }^{28-30}$

In our previous studies, we used dextran as a starting block for the fabrication of nanosystems because the base polymer of dextran is a glucan-based nontoxic material that has been approved as a plasma expander for human use by the US Food and Drug Administration. We also demonstrated that a dextran backbone can be functionalized with lipid chains to enable self-assembly to form nanoparticles. ${ }^{31}$ In different cancer model systems, dextran-based nanoparticles have been able to transport drugs and multidrug resistance (MDR1) siRNA efficiently into tumor cells to overcome drug resistance. ${ }^{32,33}$ We hypothesize that dextranbased nanoparticles could also be an ideal carrier for the delivery of therapeutic miRs into cancer cells. In the study reported here, we employed miR-199a-3p and let-7a as candidate miRs to determine the efficiency of miR delivery in osteosarcoma cells using a dextran-based self-assembling nanoparticle platform.

\section{Materials and methods}

The chemical reagents and polymers for synthesis, such as dextran, stearyl amine, cystamine, pyridine, sodium periodate, sodium cyanoborohydride, potassium sulfate, and azo-bis-isobutyronitrile were purchased from Sigma-Aldrich Co (St Louis, MO, USA). Dithiol-modified poly(ethylene glycol) (PEG-[SH]$]_{2}$, molecular weight (MW) 2,000) was purchased from SunBio, Inc. (Seoul, South Korea). Anhydrous lithium chloride ( $\mathrm{LiCl}$ ) was from Fisher Scientific (Philadelphia, PA, USA). Dehydrated dimethyl formamide (DMF) and dimethyl sulfoxide (DMSO) with molecular sieves were obtained from Acros Organics (Parsipanny, NJ, USA). Acryloyl chloride, pyridine, and other reagents and solvents were from Sigma-Aldrich Co and were used as received without further purification.

\section{Synthesis of dextran acrylate}

The synthesis of dextran acrylate was based on a previously described procedure with modifications. ${ }^{31}$ In brief, a fixed amount of dextran (MW $\sim 40 \mathrm{kDa}, 2 \mathrm{~g}$ ) was added to an $\mathrm{LiCl} / \mathrm{DMF}(4 \% \mathrm{w} / \mathrm{v}, 50 \mathrm{~mL})$ solvent mixture in a roundbottom flask $(200 \mathrm{~mL})$. The temperature of the oil bath was raised from room temperature to $120^{\circ} \mathrm{C}$ over a period of 2 hours. The resultant mixture became a homogeneous goldenyellow-colored solution. The solution was cooled to room temperature, and pyridine $(500 \mu \mathrm{L})$ was added and stirred. The reaction mixture was cooled to $0^{\circ} \mathrm{C}$ using an ice bath, and varying amounts of acryloyl chloride (1.0-1.5 molar excess) was added drop-wise using an addition funnel. The reaction was maintained at $0^{\circ} \mathrm{C}$ until total addition of acryloyl chloride was complete, over a period of 1-2 hours. The reaction was allowed to continue overnight with stirring.

The dextran acrylate obtained was precipitated in excess cold ethanol and washed three times with absolute 
ethanol. For confirmation of the formation of dextran acrylate, a small portion of the acrylate monomer was polymerized using $0.001 \%$ azo-bis-isobutyronitrile initiator in DMSO at $60^{\circ} \mathrm{C}$ for 24 hours, which resulted in formation of the acrylate polymer, confirming the reaction. Alternately, for lipid modification, the dextran acrylate was directly used as the monomer for the next step, in a one-pot synthesis.

\section{Synthesis of lipid-modified dextran}

Dextran acrylate synthesized in the previously described step was dissolved in dry DMF and stirred in a glass vial with varying amounts (5-10 mole \%) of stearyl amine and a catalyst $\left(0.01\right.$ mole $\left.\% \mathrm{AlCl}_{3}\right)$. The reaction mixture was heated to $40^{\circ} \mathrm{C}-50^{\circ} \mathrm{C}$ in an oil bath for 24 hours. The product obtained (stearyl amine-modified dextran) was precipitated and washed in cold ethanol several times to purify the product. Finally, the lipid-modified dextran derivative was dissolved in a small amount of deionized water and lyophilized to yield the pale-yellow-colored final product. The stearyl modification of dextran was confirmed by nuclear magnetic resonance spectroscopy and the percent lipid modification was estimated to be 5-7 mole $\%$.

\section{Synthesis of thiolated dextran}

The dextran backbone was oxidized based on a reported procedure.$^{34}$ In brief, $\mathrm{NaIO}_{4}$ was dissolved in $60 \mathrm{~mL}$ of deionized water. The solution was added to a solution containing $4 \mathrm{~g}$ of dextran in $30 \mathrm{~mL}$ of deionized water. The reaction was stirred in the dark for 2 hours at room temperature. At the end of the reaction, the solution was dialysed extensively against deionized water, using Spectra/Por ${ }^{\circledR}$ dialysis membranes (MW cutoff 12-14 kDa; Spectrum Labs, Rancho Dominguez, CA, USA) extensively against deionized water $(2 \mathrm{~L})$ for 4 days with several water replacements. A powdery, freeflowing sample was obtained after freeze-drying (yield $3.7 \mathrm{~g}$ [92.5\%]).

A $500 \mathrm{mg}$ portion of the oxidized dextran was dissolved in $50 \mathrm{~mL}$ of pH 5.2 buffer containing $\mathrm{K}_{2} \mathrm{SO}_{4}$ and $\mathrm{NaCNBH}_{3}$. Then, $50 \mathrm{mg}$ of cystamine was added and the mixture stirred at $40^{\circ} \mathrm{C}$ for 4 days. The product was subjected to extensive dialysis and then lyophilized to yield thiolated dextran. All compounds synthesized were routinely characterized by high-performance liquid chromatography, liquid chromatography-mass spectrometry, proton- and carbon-13 nuclear magnetic resonance spectroscopy, Elman assay, and Fourier transform infrared spectroscopy to assure chemical identity and purity.

\section{Encapsulation of microRNAs in functionalized dextran-based nanoparticles}

The miR-containing dextran nanoparticles were fabricated in a similar fashion to the siRNA-loaded dextran nanosystems reported by our group recently. ${ }^{32,33}$ Briefly, a stock solution of $5 \mathrm{mg} / \mathrm{mL}(\sim 2.5 \mathrm{mM})$ dextran thiol, $5 \mathrm{mg} / \mathrm{mL}(\sim 2.5 \mathrm{mM})$ dextran hexylamine, $5 \mathrm{mg} / \mathrm{mL}(125 \mu \mathrm{M})$ PEG-thiol, and $2 \mu \mathrm{M}$ miR solution was prepared. For obtaining a $100 \mathrm{nM}$ miR concentration, a $120 \mu \mathrm{L}$ stock solution of miR was mixed with $40 \mu \mathrm{L}$ of dextran-thiol stock solution using a vortex shaker. It was then incubated for 5 minutes. To this mixture, $40 \mu \mathrm{L}$ of dextran-hexylamine derivatives were added and incubated for another 5 minutes. Finally, $40 \mu \mathrm{L}$ of PEG-(SH) $)_{2}$ was added and incubated for another 15 minutes to form the PEGylated nanoparticles.

This method of sequential addition was used so that the miR would be entrapped in the interpenetrating dextran hydrogel network. Further, a PEG-thiol derivative was used to form intermolecular cross-linking of individual nanoparticles decorated with PEG on the surface. The final mixture was subsequently diluted with the medium and applied at the designated concentrations. Nanoparticles containing $100 \mathrm{nM}$ of nonspecific miRs were used as negative controls in the experiments.

\section{Measurement of size and zeta potentials, and transmission electron microscopy of microRNA-loaded dextran nanoparticles}

The particle size and zeta potentials of the miR-199a-3ploaded dextran nanoparticles were determined with a Zetasizer Nano S (Malvern Instruments Ltd, Malvern, UK). For light-scattering experiments, the samples were measured at fixed angle of $90^{\circ}$ at $25^{\circ} \mathrm{C}$. The scattering intensity was adjusted in the range of 50-200 kcps by diluting the samples with phosphate-buffered saline (PBS). For zeta potentials, the default parameters of dielectric constant, refractive index, and viscosity of water were used based on the electrophoretic mobility of the nanoparticles. Transmission electron micrographs of nanoparticles were obtained using a JEM1000 Ultra High Voltage Electron Microscope (JEOL Ltd, Tokyo, Japan).

\section{Cell culture and reagents}

Osteosarcoma cell lines, KHOS and U-2OS, were cultured with Dulbecco's Modified Eagle's Medium (DMEM), supplemented with $10 \%$ fetal bovine serum, $100 \mathrm{U} / \mathrm{mL}$ penicillin, and $100 \mathrm{U} / \mathrm{mL}$ streptomycin (Thermo Fisher Scientific, Waltham, 
MA, USA). The cells were incubated at $37^{\circ} \mathrm{C}$ in a $5 \% \mathrm{CO}_{2}, 95 \%$ air atmosphere. The fluorescent-labeled AF488-miR-199a-3p (sequences: 5'-ACAGUAGUCUGCACAUUGGUUA-3', green color) and A546-labeled let-7a (sequences: 5'-UGAGGUAGUAGGUUGUAUAGUU-3', red color) were purchased from Qiagen NV (Venlo, the Netherlands). The unlabeled miR-199a-3p, let-7a, nonspecific miR mimic, and Life Technologies Lipofectamine ${ }^{\circledR}$ RNAiMAX were purchased from Thermo Fisher Scientific.

\section{Determination of microRNA cellular uptake by dextran nanoparticles}

Evaluation of the cellular uptake of miR was based on a previously described procedure. ${ }^{35}$ In brief, osteosarcoma cell lines U-2OS and KHOS were seeded at density of $1 \times 10^{5}$ cells/well in 24-well plates (three wells per cell line). After 24 hours' incubation, $100 \mathrm{nM}$ AF488-miR-199a-3p, or A546let-7a encapsulated in dextran-based nanoparticles or transfected with Lipofectamine ${ }^{\circledR}$ RNAiMAX (Invitrogen); or 100 nM AF488-miR-199a-3p or A546-let-7a alone without nanoparticles were added into each well of cultured U-2OS and KHOS cell plate.

The efficiency of cellular uptake was examined at 2, 4, and 24 hours post-transfection. To determine the subcellular distributions of miR encapsulated in dextran-based nanoparticles, U-2OS cells transfected with $100 \mathrm{nM}$ A546-let-7a encapsulated in dextran-based nanoparticles were visualized 24 hours post-transfection under a Nikon Eclipse Ti-U fluorescence microscope (Nikon Instruments, Inc., Melville, NY, USA) equipped with a SPOT RT ${ }^{\text {TM }}$ digital camera (Diagnostic Instruments, Inc., Sterling Heights, MI, USA). Just prior to microscopy, the cells were incubated with $1 \mu \mathrm{g} / \mathrm{mL}$ Hoechst 33342 (Thermo Fisher Scientific) for 1 minute for nuclear counterstaining. Then, the cells were washed with PBS and visualized under microscope. Fluorescence intensity and cellular localization was analyzed in triplicate at different fields of view randomly.

\section{MicroRNA real-time polymerase chain reaction assay}

Osteosarcoma U-2OS cells were transfected with $100 \mathrm{nM}$ of miR-199a-3p or let-7a encapsulated in dextran-based nanoparticles, or tranfected with Lipofectamine ${ }^{\circledR}$ RNAiMAX, or miR-199a-3p, or let-7a alone. The total RNA (including miRs) was extracted using an miRNANeasy Mini Kit (Qiagen NV) by following the manufacturer's instructions 48 hours posttransfection. The concentration of RNA was evaluated using a Beckman DU-640 spectrophotometer (Beckman Instruments,
Inc., Fullerton, CA, USA). The quality of RNA was determined by $1 \%$ formaldehyde-agarose gel electrophoresis. An Applied Biosystems ${ }^{\circledR}$ TaqMan ${ }^{\circledR}$ MicroRNA Reverse Transcription Kit (Thermo Fisher Scientific) was used for quantification of miR expression. Complementary DNA reverse transcription was performed from total miR samples using specific miR primers of miR-199a-3p or let-7a from the TaqMan MicroRNA Assay Kits. Quantitative real-time polymerase chain reaction (PCR) was performed on an Applied Biosystems StepOne ${ }^{\text {TM }}$ RealTime PCR System (Thermo Fisher Scientific) for 40 cycles (of $95^{\circ} \mathrm{C}$ for 15 seconds, $60^{\circ} \mathrm{C}$ for 15 seconds, and $72^{\circ} \mathrm{C}$ for 30 seconds). Applied Biosystems RNU48 miR (Thermo Fisher Scientific) was used as endogenous control of miR expression. Untreated cells were used as reference samples. The relative gene-expression levels were then normalized by RNU48 and calculated using the Comparative CT $\left(2^{\wedge}-\Delta \Delta \mathrm{Ct}\right)$ method. The relative gene-expression levels were $\log 10$ transformed prior to analysis. All experiments were carried out in triplicate.

\section{Western blot analysis}

Osteosarcoma U-2OS and KHOS cells were transfected with $100 \mathrm{nM}$ of miR-199a-3p encapsulated in dextran-based nanoparticles or transfected with Lipofectamine ${ }^{\circledR}$ RNAiMAX; or transfected with $100 \mathrm{nM}$ of Met siRNA (positive control) with Lipofectamine ${ }^{\circledR}$ RNAiMAX, nanoparticle or miR-199a-3p alone. Proteins were extracted from the transfected cells using $1 \times$ radioimmunoprecipitation assay (RIPA) lysis buffer (Upstate Biotechnology, Lake Placid, NY, USA). The protein concentration was determined using protein assay reagents and standard protocols (Bio-Rad Laboratories, Hercules, CA, USA). Briefly, $25 \mu \mathrm{g}$ of total protein was processed on Life Technologies NuPAGE ${ }^{\circledR} \%-12 \%$ BisTris gel (Thermo Fisher Scientific) and transferred to a pure nitrocellulose membrane (Bio-Rad Laboratories, Hercules, CA). After transfer, the membranes were blocked with Odyssey ${ }^{\circledR}$ Blocking Buffer (LI-COR Biosciences, Lincoln, NE, USA), and then incubated in primary antibodies buffer (primary antibodies, Odyssey Blocking Buffer, 0.1\% Tween ${ }^{\circledR} 20$ ) overnight at $4^{\circ} \mathrm{C}$. Three primary antibodies, insulin-like growth factor 1 receptor (IGF-1R), mammalian target of rapamycin (mTOR), and Met were purchased from Cell Signaling Technology, Inc. (Beverly, MA, USA). The following day, membranes were washed four times for 5 minutes in Tris-buffered saline and Tween 20 (TBST). Subsequently, membranes were incubated in secondary antibodies IRDye ${ }^{\circledR}$ 680LT Goat Anti-Mouse lgG or IRDye 680LT Goat Anti-Rabbit lgG (LI-COR 
Biosciences) plus Odyssey Blocking Buffer and 0.1\% Tween 20 at 1:20,000 dilution for 1 hour. Finally, the membranes were scanned on an Odyssey CLx imaging system, model:Ody-3086 (LI-COR Inc., Lincoln, NE).

\section{Cell proliferation assay}

The effects of overexpression of miR delivered by dextranbased nanoparticles on osteosarcoma cell proliferation were determined by 3(4,5-dimethylthiazol-2-yl)-2,5-diphenyltetrazolium bromide (MTT) assay. The cells were seeded at a density of 2,000 cells/well in 96-well culture plates and incubated for 24 hours at $37^{\circ} \mathrm{C}$ prior to transfection. The following day, cells were transfected with miR-199a-3p or let-7a encapsulated in dextran-based nanoparticles, or transfected with miR-199a-3p or let-7a Lipofectamine ${ }^{\circledR}$ RNAiMAX. After 96 hours, $20 \mathrm{~mL}$ of MTT solution $(5 \mathrm{mg} / \mathrm{mL}$ in PBS) was added to each well. Samples were further incubated for 4 hours. The resulting formazan product was dissolved with HCL-isopropanol and the absorbance was read on a SpectraMax ${ }^{\circledR}$ microplate spectrophotometer (Molecular Devices, LLC, Sunnyvale, CA, USA) at a wavelength of $490 \mathrm{~nm}$. Experiments were carried out in triplicates. Cell growth curves were fitted with use of GraphPad PRISM4 software (GraphPad Software, Inc., La Jolla, CA).

\section{Statistical analysis}

Student's $t$-test was used to compare the differences between groups. Results are given as mean \pm standard deviation and results with $P<0.05$ are considered statistically significant.

\section{Results}

\section{Characterization of dextran nanoparticles encapsulated with microRNA}

We previously developed a polymeric self-assembling nanoparticle system based on a functionalized dextran macrostructure to stably encapsulate siRNA molecules. In the current study, we used a similar method to encapsulate miRs. The selected miRs were incubated with the lipid-modified dextran (dextran-hexyl amine) derivative in deionized water at room temperature to form nanoparticles by self-assembly. Further, a thiolated-dextran derivative and a PEGylated-dextran derivative were sequentially mixed with the miRs containing lipid-modified dextran to form intramolecular disulfide cross-linking of the dextran hydrogel network with protruding PEG chains to stabilize the individual nanoparticles. The method of sequential addition helped in the formation of stable nanoparticles with good miR entrapment.

The mean Z-average particle size (nm) of the miR-199a$3 \mathrm{p}$-loaded nanoparticles was $351.6 \pm 2.5 \mathrm{~nm}$ (Figure 1A), as

C

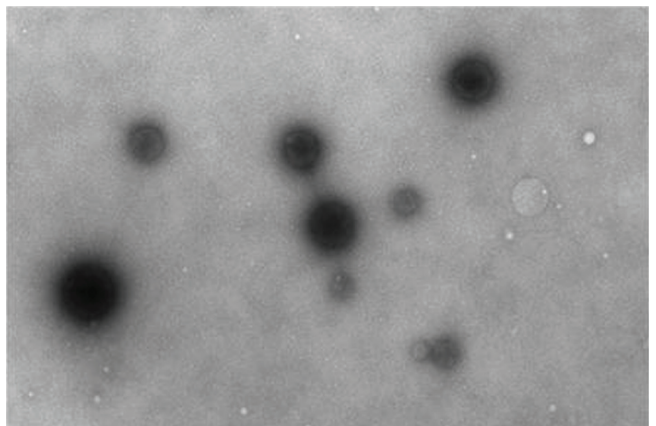

B

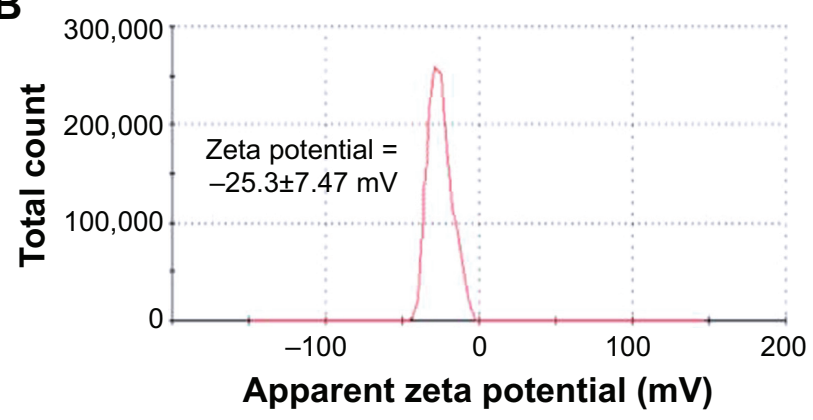

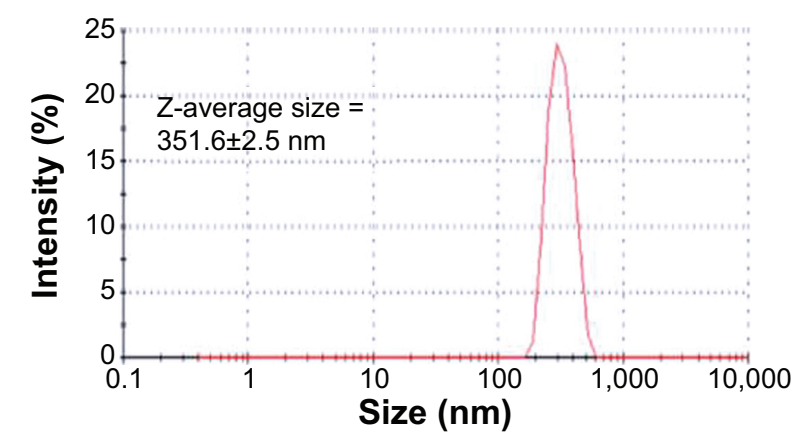

Apparent zeta potential (mV)

Figure I Characterization of microRNA encapsulated dextran nanoparticles.

Notes: (A) Z -average particles size $(\mathrm{nm})$ and $(\mathbf{B})$ apparent zeta potentials $(\mathrm{mV})$ was determined by dynamic light scattering (Malvern Instuments Inc., Westborough, MA) and (C) transmission electron microscopy of miR-199q-3p-loaded dextran nanoparticles was recorded using a JEM-I000 electron microscope (JEOL Ltd, Tokyo, Japan). 
determined by dynamic light scattering measurement, and the zeta potential was $-25.3 \pm 7.47 \mathrm{mV}$ (Figure 1B). The size of miR-199a-3p-loaded dextran nanoparticles was also characterized and confirmed by transmission electron micrograph (Figure 1C). The miR particles were stable at room temperature and there was no significant change in particle size or zeta potential from storage (for up to 1 week at $4^{\circ} \mathrm{C}$ ) in PBS. In addition the miR-loaded nanoparticels were amenable to lyophilization and stored at $4^{\circ} \mathrm{C}$ for prolonged periods.

\section{Cellular uptake of microRNAs transfected with dextran-based nanoparticles}

To assess the transfection efficacy of miR-loaded dextran nanoparticles on osteosarcoma cells, the cellular uptake of fluorescently labeled miR was assessed using a fluorescence microscope. Cells transfected with fluorescently labeled (AF488)-miR-199a-3p or (A546)-let-7a mixed with Lipofectamine RNAiMAX were used as positive controls, and cells treated with AF488-miR-199a-3p or A546-let-7a alone were used as negative controls.

The results demonstrated that the dextran nanoparticles could efficiently transfect AF488-miR-199a-3p into U-2OS cells after 2 hours' incubation, and the fluorescence signal increased with increasing duration of incubation time (Figure 2A-C). Interestingly, in comparison with miR transfected using the commercial transfection agent (Lipofectamine; Figure 2D-F), dextran-based nanoparticles did not exhibit any apparent toxicity to the tumor cells and could promote miRs' transfection into cells for longer duration of time. Similar results were observed for U-2OS cells transfected with A546let-7a miRs loaded in dextran nanoparticles (Figure S1).

In addition, subcellular distributions of A546-let-7a in dextran-based nanoparticle-transfected cells were also analyzed under fluorescence microscope. The results showed clear cytoplasmic distribution of fluorescently labeled A546let-7a miR. Similar to the observation with cells treated with miR-199a-3p loaded in dextran nanoparticles, cells transfected with dextran nanoparticles encapsulated with A546-let-7a miR also exhibited negligible toxicity compared with cells transfected with miRs using Lipofectamine RNAiMAX (Figure S2).

These results demonstrate the advantages of using nontoxic dextran-based nanoparticles for the efficient transfection of miRs into cancer cells.
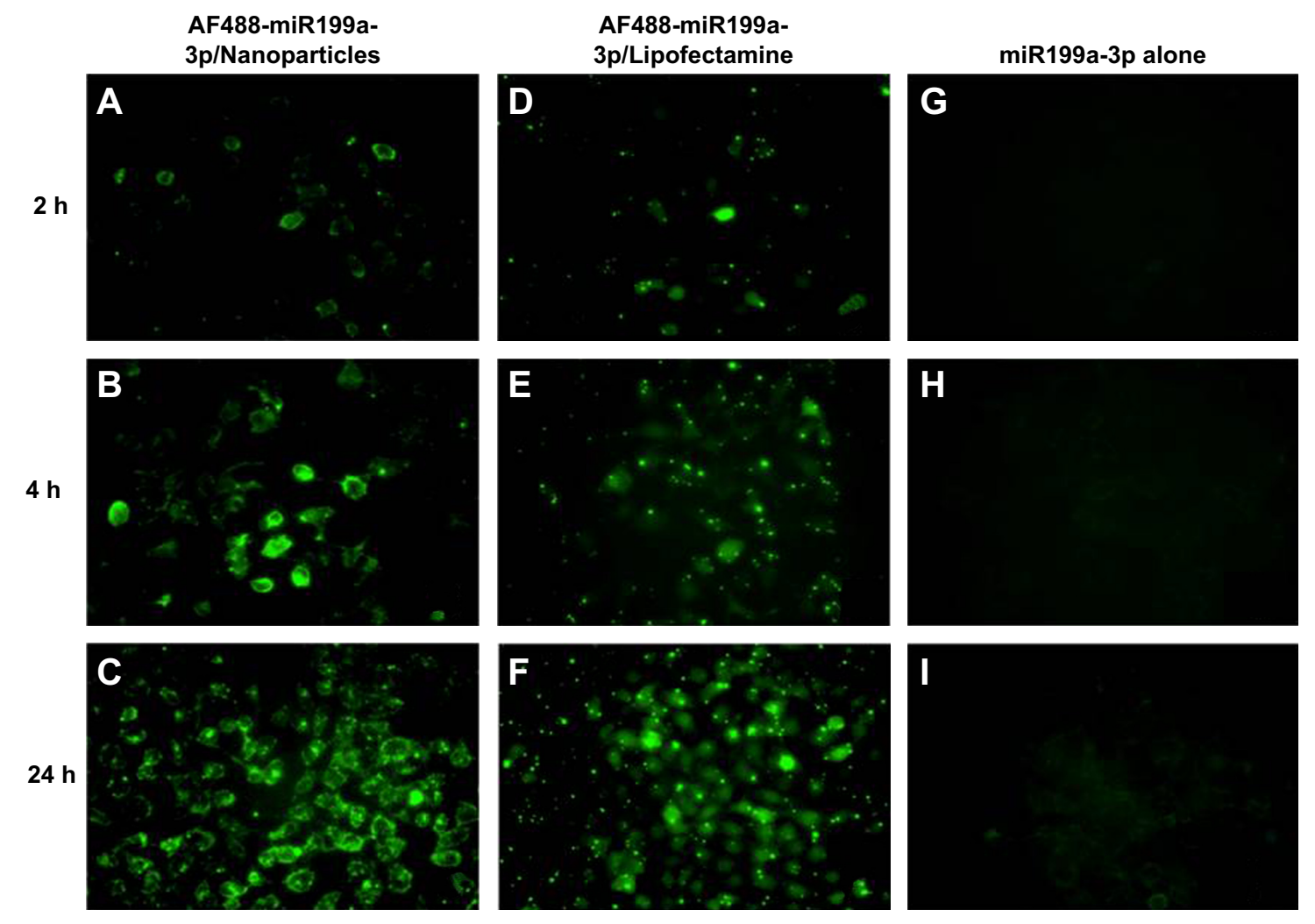

Figure 2 Intracellular trafficking of microRNA encapsulated dextran nanoparticles in osteosarcoma cells.

Notes: Cellular uptake of fluorescently labeled AF488-miR-199a-3p encapsulated with dextran nanoparticles. U-2OS cells were transfected with AF488-miRI99a-3p (green) encapsulated with dextran nanoparticle $(\mathbf{A}-\mathbf{C})$, with LipofectamineTM RNAiMax (D-F), or AF488-miRI99a-3p alone (G-I). The cells were visualized by florescence microscopy at 2, 4, and 24 hours after transfection. 


\section{MicroRNA expression in dextran nanoparticle-transfected cells}

After fluorescence microscopy showed that dextran nanoparticles are able to deliver fluorescently labeled miR into osteosarcoma cells, the expression of miR was further determined by real-time PCR with total RNA isolated from the osteosarcoma cells, as described in the "Materials and methods" section. The results indicate that miR-199a-3p (Figure 3A) and let-7a miR (Figure 3B) were significantly expressed in dextran nanoparticle-transfected osteosarcoma cells. The expression level of let-7a in U-2OS cells was also evaluated and confirmed at 24,48 , and 72 hours posttransfection (Figure 3C).

\section{MicroRNA-targeted gene protein expression in dextran nanoparticle- transfected cells}

It has been shown that mTOR and Met are direct targets of miR-199a-3p. ${ }^{11,13-15}$ To assess the functional role of miR delivered by dextran nanoparticles, the expression of target gene proteins in miR-199a-3p nanoparticle-transfected cells was measured using Western blotting. Relative mTOR and Met expression levels from the Western blot were analyzed by densitometry. The results show that nanoparticles encapsulated with miR-199a-3p can inhibit the expression of mTOR and Met in both U-2OS (Figure 4A and C) and KHOS (Figure 4B and D) osteosarcoma cell lines. As a control, Met siRNA can also decrease the expression of Met and have no effect on the expression of mTOR in both U-2OS and KHOS cell lines, as expected (Figure 4A-D).

\section{Effect on osteosarcoma cell growth and proliferation in dextran nanoparticle- transfected cells}

To determine whether transfection of dextran nanoparticles encapsulated miR-199a-3p or let-7a is able to alter the rate of cell proliferation and growth, osteosarcoma U-2OS and KHOS cells were transfected with miR-199a-3p or let-7a encapsulated with dextran-based nanoparticles, or transfected with Lipofectamine ${ }^{\circledR}$ RNAiMAX. Non-specific miR mimic loaded in dextran based nanoparticles or mixed with Lipofectamine $^{\circledR}$ RNAiMAX were used as controls. MTT assay showed that the delivery of miR-199a-3p (Figure 5A and $\mathrm{C}$ ) or let-7a (Figure 5B and D) using dextran nanoparticles significantly decreased cell proliferation in both U-2OS (Figure 5A and B) and KHOS (Figure 5C and D) osteosarcoma cell lines. Furthermore, the inhibition of cell growth was dependent on the dose of miR, with the $100 \mathrm{~nm}$ concentration of miR exhibiting more potent inhibition than the $50 \mathrm{~nm}$ dose (Figure 5).

\section{Discussion}

miRs have been identified as playing a pivotal role in the regulation of key genes implicated in cancer maintenance, progression, and drug resistance. ${ }^{36}$ More recently, a number of research studies, both laboratory and clinic based, have
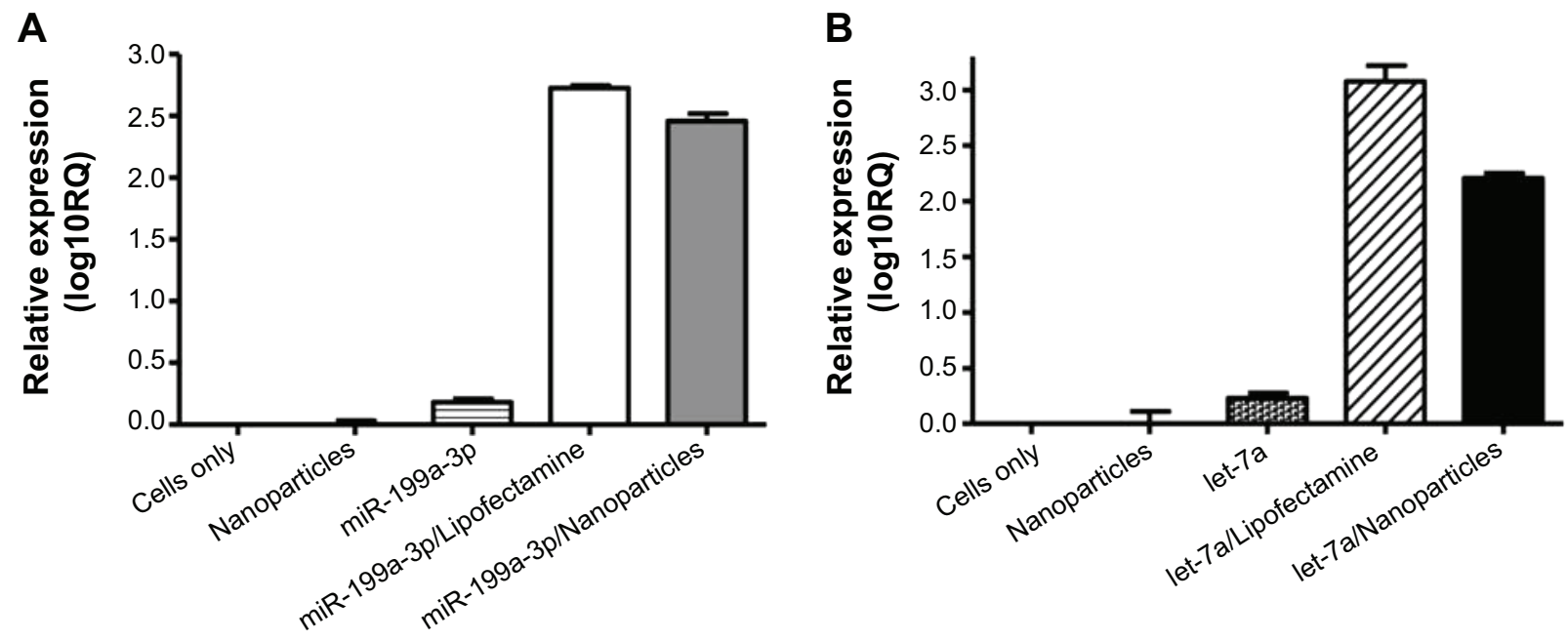

Figure 3 Expression levels of microRNAs transfected by dextran nanoparticles.

Notes: Expression of miR-199a-3p or let-7a in osteosarcoma cells transfected by dextran nanoparticles. U-2OS cells were transfected with miRI99a-3p (A) or I00 nM let-7a (B) encapsulated with dextran nanoparticle, or transfected with with Lipofectamine ${ }^{T M}$ RNAiMax. The total RNA (including miRs) was extracted after 48 hours post transfection. Expressions of miR-199a-3p or let-7a were determined by TaqMan MicroRNA Assay kits.

Abbreviation: RQ, relative quantity. 


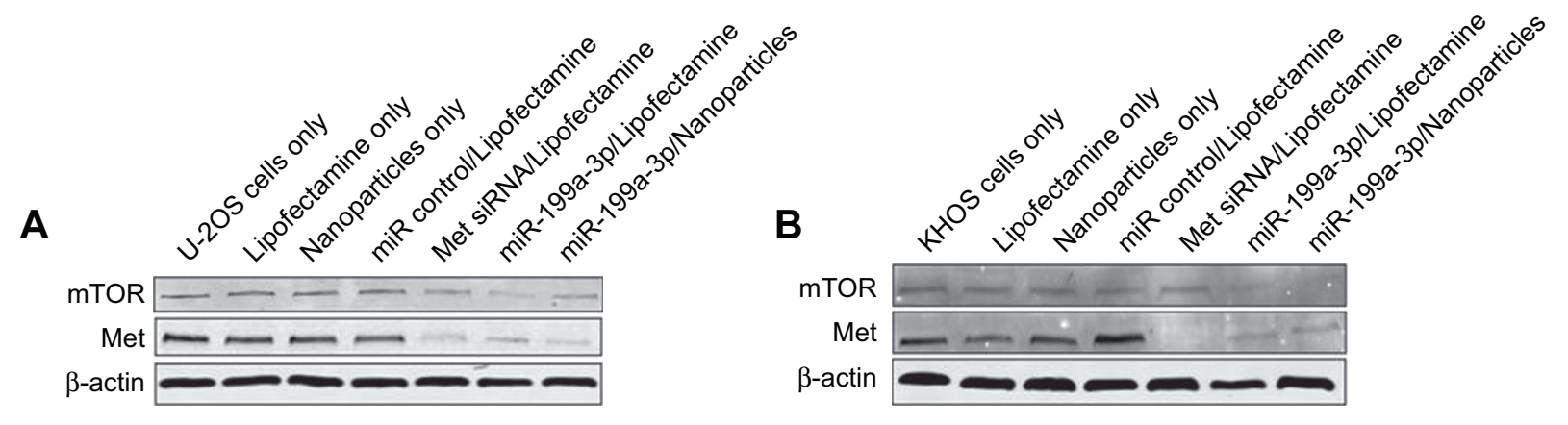

C

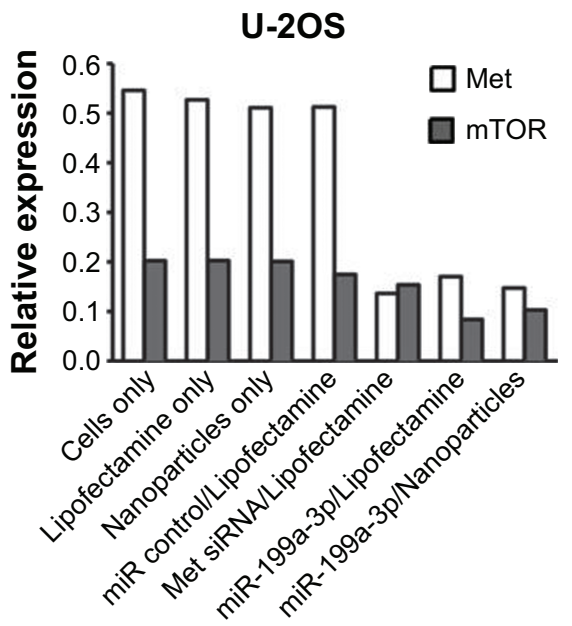

D

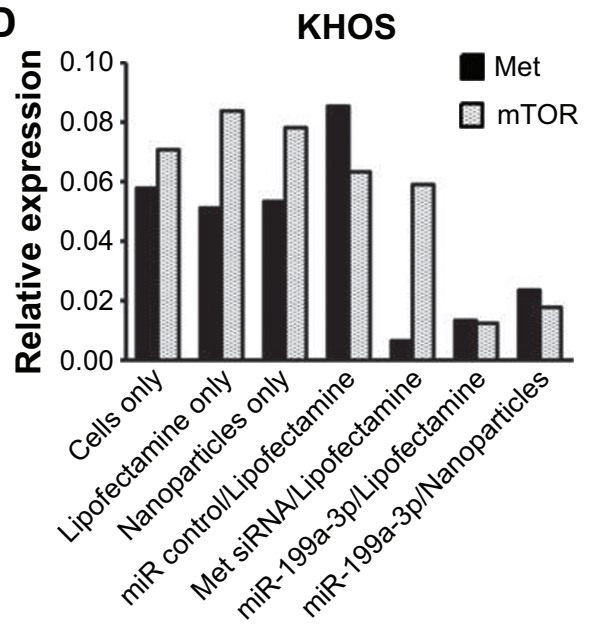

Figure 4 Protein expression levels in osteosarcoma cells transfected with microRNAs delivered using dextran nanoparticles.

Notes: Protein expression in miR-199a-3p dextran nanoparticle transfected osteosarcoma cells. U-2OS (A) and KHOS (B) cells were transfected with miR-I99a-3p encapsulated with dextran-based nanoparticles, or transfected with Lipofectamine ${ }^{\circledR}$ RNAiMAX. The expression of mTOR and Met were determined by Western blots, and the data were analyzed by densitometry $(\mathbf{C}$ and $\mathbf{D})$.

Abbreviation: $\beta$-actin, beta-actin.

reported on the implications of miRs in osteosarcoma. ${ }^{7,8,10,11}$ Several research groups have shown that the expressions of miRs in osteosarcoma cells and in normal cells are significantly different. Although these studies suggest that miRs have important roles in the development of osteosarcoma, the potential development of miRs as a therapeutic agent is largely unexplored. In this study, we demonstrated the exogenous transfection of miR-199a-3p into osteosarcoma cells using a dextran nanoparticle-based delivery system. The miR-199a-3p-encapsulated nanoparticles showed high miR transfection efficiency, significantly increased miR199a-3p expression, and decreased mTOR and Met expression, resulting in a suppression effect on osteosarcoma cell proliferation and growth. The dextran nanoparticles also showed good delivery efficiency for let-7a, another tumorsuppressing miR.

Our group and others have previously shown that dextran-based nanoparticles are able to efficiently deliver both the chemotherapy drug doxorubicin as well as siRNA to osteosarcoma cells with no apparent toxicity. ${ }^{31-33,37} \mathrm{We}$ also previously found that dextran-based nanoparticles can efficiently transport siRNA into tumor cells. ${ }^{32,33}$ More recently, cationized dextran nanoparticles encapsulated with C-X-C chemokine receptor type 4 (CXCR4) siRNA have been shown to significantly downregulate CXCR4 expression in colorectal cancer, both in vitro and in vivo. ${ }^{38}$ CXCR4 is highly overexpressed in various types of cancers. A dextran-based nanosystem containing epidermal growth factor receptor siRNA has also been successfully developed. ${ }^{39}$ Furthermore, folates have been introduced as an active tumortargeting moiety to the hydroxyl groups of dextran to form dextran-siRNA conjugates. The data show that these dextranbased nanosystems are very efficiently targeted to cancer cells and induce sequence-specific gene silencing. ${ }^{40}$

Our current study demonstrates - for the first time, as far as we are aware - that dextran nanoparticles are also capable of delivering miRs- with an efficiency similar to delivering siRNAs to cancer cells. By using two different 

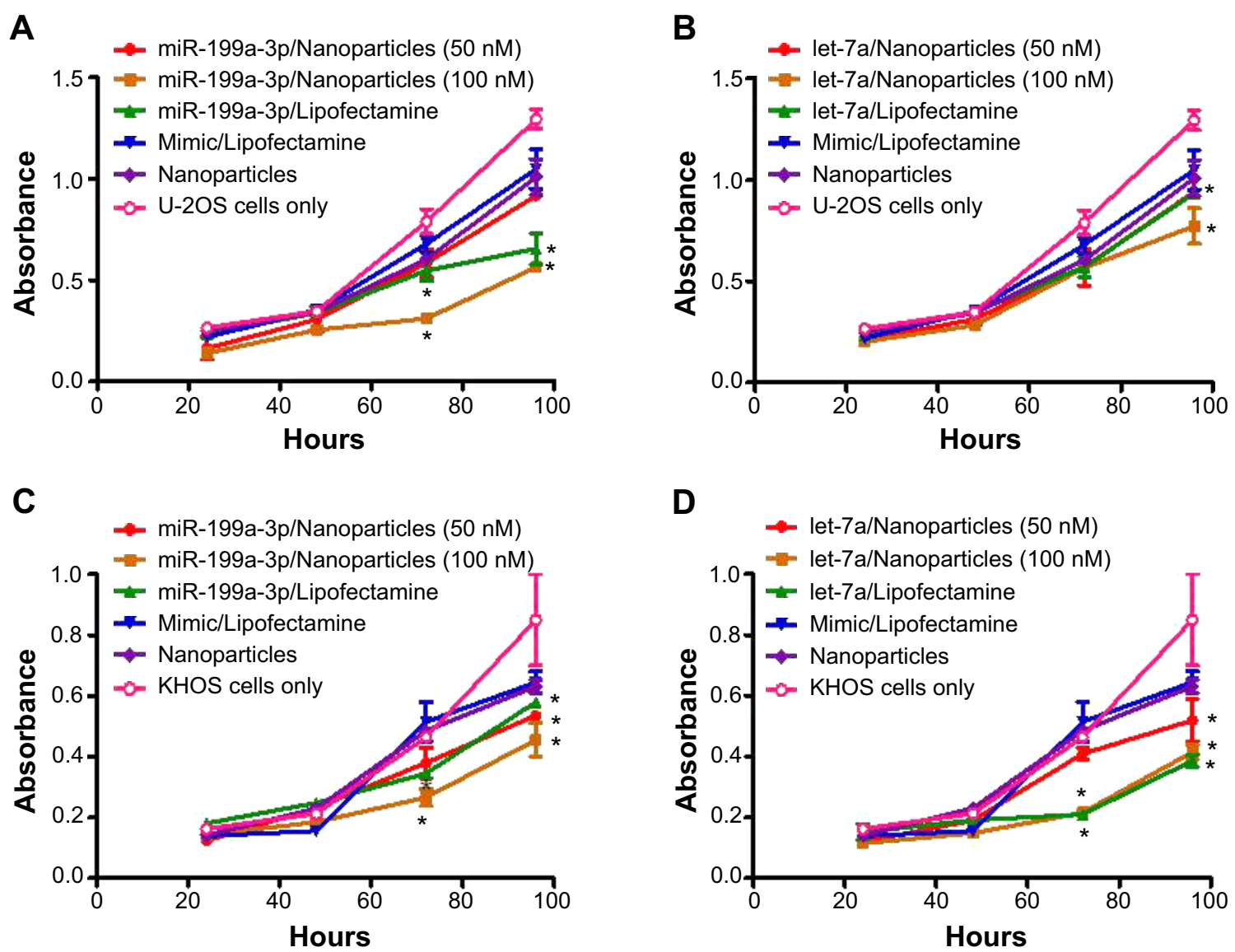

Figure 5 Osteosarcoma cell growth inhibition using microRNA encapsulated dextran nanoparticles.

Notes: Effect on osteosarcoma cell growth and proliferation in dextran nanoparticle transfected cells. U-2OS (A and B) and KHOS (C and D) cells were transfected with miR-199a-3p (A and C) or let-7a (B and $\mathbf{D})$ encapsulated with dextran-based nanoparticles, or transfected with Lipofectamine ${ }^{\circledR}$ RNAiMAX. MTT assay was performed by measuring the absorbance at a wavelength of $490 \mathrm{~nm}$. Data are represented as means \pm SD and analyzed using Student's $t$-test. *indicates $P<0.05$.

Abbreviation: MTT, 3-(4,5-dimethylthiazol-2-yl)-2,5-diphenyltetrazolium bromide.

fluorescently labeled miRs, AF488-miR-199a-3p (green) and A546-let-7a (red) encapsulated in dextran nanoparticles, we tracked the intracellular uptake of miRs using fluorescence microscopy. Both AF488-miR-199a-3p and A546-let-7a could be visualized in the cytoplasm of osteosarcoma cells after transfection. miR-199a-3p and let-7a miRs were significantly expressed in the different dextran nanoparticle-transfected osteosarcoma cells, as demonstrated by real-time PCR.

More studies are needed to test the delivery efficiency of miR in vivo using these dextran nanoparticles. These studies will represent the first step toward the advancement of miR- and nanotechnology-based therapy strategies in combating osteosarcoma. In addition, the ability of dextran nanoparticles to transfect both miRs and siRNAs, as well as chemotherapy drugs, into cancer cells may represent a favorable multi-pronged approach to treating virulent diseases such as multidrug-resistant tumors.

\section{Conclusion}

This study demonstrates the utility of using dextran-based nanoparticles to deliver miRs in cancer cells. Dextranbased nanoparticles portend to be a promising platform to overcome the current limitations of miR delivery for the treatment of human cancers. However, the translation of these nanosystems for clinical use can be determined only after thorough in vivo evaluation in appropriate animal tumor models. The feasibility of such studies is underway in our laboratory.

\section{Acknowledgments}

This study was supported in part by the National Cancer Institute's Alliance for Nanotechnology in Cancer through the Cancer Nanotechnology Platform Partnership (grant number U01-CA15145). Support was also received from the Gattegno and Wechsler funds, and in the form of a grant from the Sarcoma Foundation of America. 


\section{Disclosure}

The authors report no conflicts of interest in this work.

\section{References}

1. Arndt CA, Crist WM. Common musculoskeletal tumors of childhood and adolescence. N Engl J Med. 1999;341(5):342-352.

2. Bielack SS, Marina N, Ferrari S, et al. Osteosarcoma: the same old drugs or more? J Clin Oncol. 2008;26(18):3102-3103; author reply 3104-3105.

3. Gill J, Ahluwalia MK, Geller D, Gorlick R. New targets and approaches in osteosarcoma. Pharmacol Ther. 2013;137(1):89-99.

4. Geller DS, Gorlick R. Osteosarcoma: a review of diagnosis, management, and treatment strategies. Clin Adv Hematol Oncol. 2010; 8(10):705-718.

5. Chou AJ, Geller DS, Gorlick R. Therapy for osteosarcoma: where do we go from here? Paediatr Drugs. 2008;10(5):315-327.

6. Chou AJ, Gorlick R. Chemotherapy resistance in osteosarcoma: current challenges and future directions. Expert Rev Anticancer Ther. 2006;6(7):1075-1085.

7. Subramanian $\mathrm{S}$, Lui WO, Lee CH, et al. MicroRNA expression signature of human sarcomas. Oncogene. 2008;27(14):2015-2026.

8. Jones KB, Salah Z, Del Mare S, et al. miRNA signatures associate with pathogenesis and progression of osteosarcoma. Cancer Res. 2012;72(7):1865-1877.

9. Heneghan HM, Miller N, Kerin MJ. MiRNAs as biomarkers and therapeutic targets in cancer. Curr Opin Pharmacol. 2010;10(5):543-550.

10. Kobayashi E, Hornicek FJ, Duan Z. MicroRNA Involvement in Osteosarcoma. Sarcoma. 2012;2012:359739.

11. Duan Z, Choy E, Harmon D, et al. MicroRNA-199a-3p is downregulated in human osteosarcoma and regulates cell proliferation and migration. Mol Cancer Ther. 2011;10(8):1337-1345.

12. Duan Z, Choy E, Nielsen GP, et al. Differential expression of microRNA (miRNA) in chordoma reveals a role for miRNA-1 in Met expression. J Orthop Res. 2010;28(6):746-752.

13. Fornari F, Milazzo M, Chieco P, et al. MiR-199a-3p regulates mTOR and c-Met to influence the doxorubicin sensitivity of human hepatocarcinoma cells. Cancer Res. 2010;70(12):5184-5193.

14. Henry JC, Park JK, Jiang J, et al. miR-199a-3p targets CD44 and reduces proliferation of CD44 positive hepatocellular carcinoma cell lines. Biochem Biophys Res Commun. 2010;403(1):120-125.

15. Wu D, Huang HJ, He CN, Wang KY. MicroRNA-199a-3p regulates endometrial cancer cell proliferation by targeting mammalian target of rapamycin (mTOR). Int J Gynecol Cancer. 2013;23(7):1191-1197.

16. Yanaihara N, Caplen N, Bowman E, et al. Unique microRNA molecular profiles in lung cancer diagnosis and prognosis. Cancer Cell. 2006;9(3): 189-198.

17. Dong Q, Meng P, Wang T, et al. MicroRNA let-7a inhibits proliferation of human prostate cancer cells in vitro and in vivo by targeting E2F2 and CCND2. PloS One. 2010;5(4):e10147.

18. Jakymiw A, Patel RS, Deming N, et al. Overexpression of dicer as a result of reduced let-7 MicroRNA levels contributes to increased cell proliferation of oral cancer cells. Genes Chromosomes Cancer. 2010;49(6):549-559.

19. Chen Y, Jacamo R, Konopleva M, Garzon R, Croce C, Andreeff M. CXCR4 downregulation of let-7a drives chemoresistance in acute myeloid leukemia. J Clin Invest. 2013;123(6):2395-2407.

20. Yu CC, Chen YW, Chiou GY, et al. MicroRNA let-7a represses chemoresistance and tumourigenicity in head and neck cancer via stem-like properties ablation. Oral Oncol. 2011;47(3):202-210.
21. Kim SJ, Shin JY, Lee KD, et al. MicroRNA let-7a suppresses breast cancer cell migration and invasion through downregulation of C-C chemokine receptor type 7. Breast Cancer Res. 2012;14(1):R14.

22. Wang M, Hu Y, Amatangelo MD, Stearns ME. Role of ribosomal protein RPS2 in controlling let-7a expression in human prostate cancer. Mol Cancer Res. 2011;9(1):36-50.

23. Akao Y, Nakagawa Y, Naoe T. let-7 microRNA functions as a potential growth suppressor in human colon cancer cells. Biol Pharm Bull. 2006;29(5):903-906.

24. Bravo V, Rosero S, Ricordi C, Pastori RL. Instability of miRNA and cDNAs derivatives in RNA preparations. Biochem Biophys Res Commun. 2007;353(4):1052-1055.

25. Shukla GC, Haque F, Tor Y, et al. A boost for the emerging field of RNA nanotechnology. ACS Nano. 2011;5(5):3405-3418.

26. Chen Y, Zhu X, Zhang X, Liu B, Huang L. Nanoparticles modified with tumor-targeting scFv deliver siRNA and miRNA for cancer therapy. Mol Ther. 2010;18(9):1650-1656.

27. Crew E, Tessel MA, Rahman S, et al. MicroRNA conjugated gold nanoparticles and cell transfection. Anal Chem. 2012;84(1):26-29.

28. Muthiah M, Park IK, Cho CS. Nanoparticle-mediated delivery of therapeutic genes: focus on miRNA therapeutics. Expert Opin Drug Deliv. 2013;10(9):1259-1273.

29. Kesharwani P, Gajbhiye V, Jain NK. A review of nanocarriers for the delivery of small interfering RNA. Biomaterials. 2012;33(29): 7138-7150.

30. Shen H, Sun T, Ferrari M. Nanovector delivery of siRNA for cancer therapy. Cancer Gene Ther. 2012;19(6):367-373.

31. Susa M, Iyer AK, Ryu K, et al. Doxorubicin loaded Polymeric Nanoparticulate Delivery System to overcome drug resistance in osteosarcoma. BMC Cancer. 2009;9:399.

32. Susa M, Iyer AK, Ryu K, et al. Inhibition of ABCB1 (MDR1) expression by an siRNA nanoparticulate delivery system to overcome drug resistance in osteosarcoma. PloS One. 2010;5(5):e10764.

33. Kobayashi E, Iyer AK, Hornicek FJ, Amiji MM, Duan Z. Lipidfunctionalized dextran nanosystems to overcome multidrug resistance in cancer: a pilot study. Clin Orthop Relat Res. 20B;471(3):915-925.

34. Martwiset S, Koh AE, Chen W. Nonfouling characteristics of dextrancontaining surfaces. Langmuir. 2006;22(19):8192-8196.

35. Cubillos-Ruiz JR, Sempere LF, Conejo-Garcia JR. Good things come in small packages: Therapeutic anti-tumor immunity induced by microRNA nanoparticles. Oncoimmunology. 2012;1(6):968-970.

36. Croce CM. Causes and consequences of microRNA dysregulation in cancer. Nat Rev Genet. 2009;10(10):704-714.

37. Sun K, Wang J, Zhang J, Hua M, Liu C, Chen T. Dextran-g-PEI nanoparticles as a carrier for co-delivery of adriamycin and plasmid into osteosarcoma cells. Int J Biol Macromol. 2011;49(2):173-180.

38. Abedini F, Hosseinkhani H, Ismail M, et al. Cationized dextran nanoparticle-encapsulated CXCR4-siRNA enhanced correlation between CXCR4 expression and serum alkaline phosphatase in a mouse model of colorectal cancer. Int J Nanomedicine. 2012;7:4159-4168.

39. Cho HJ, Chong S, Chung SJ, Shim CK, Kim DD. Poly-L-arginine and dextran sulfate-based nanocomplex for epidermal growth factor receptor (EGFR) siRNA delivery: its application for head and neck cancer treatment. Pharm Res. 2012;29(4):1007-1019.

40. Kim JS, Oh MH, Park JY, Park TG, Nam YS. Protein-resistant, reductively dissociable polyplexes for in vivo systemic delivery and tumor-targeting of siRNA. Biomaterials. 2013;34(9):2370-2379. 


\section{Supplementary materials}

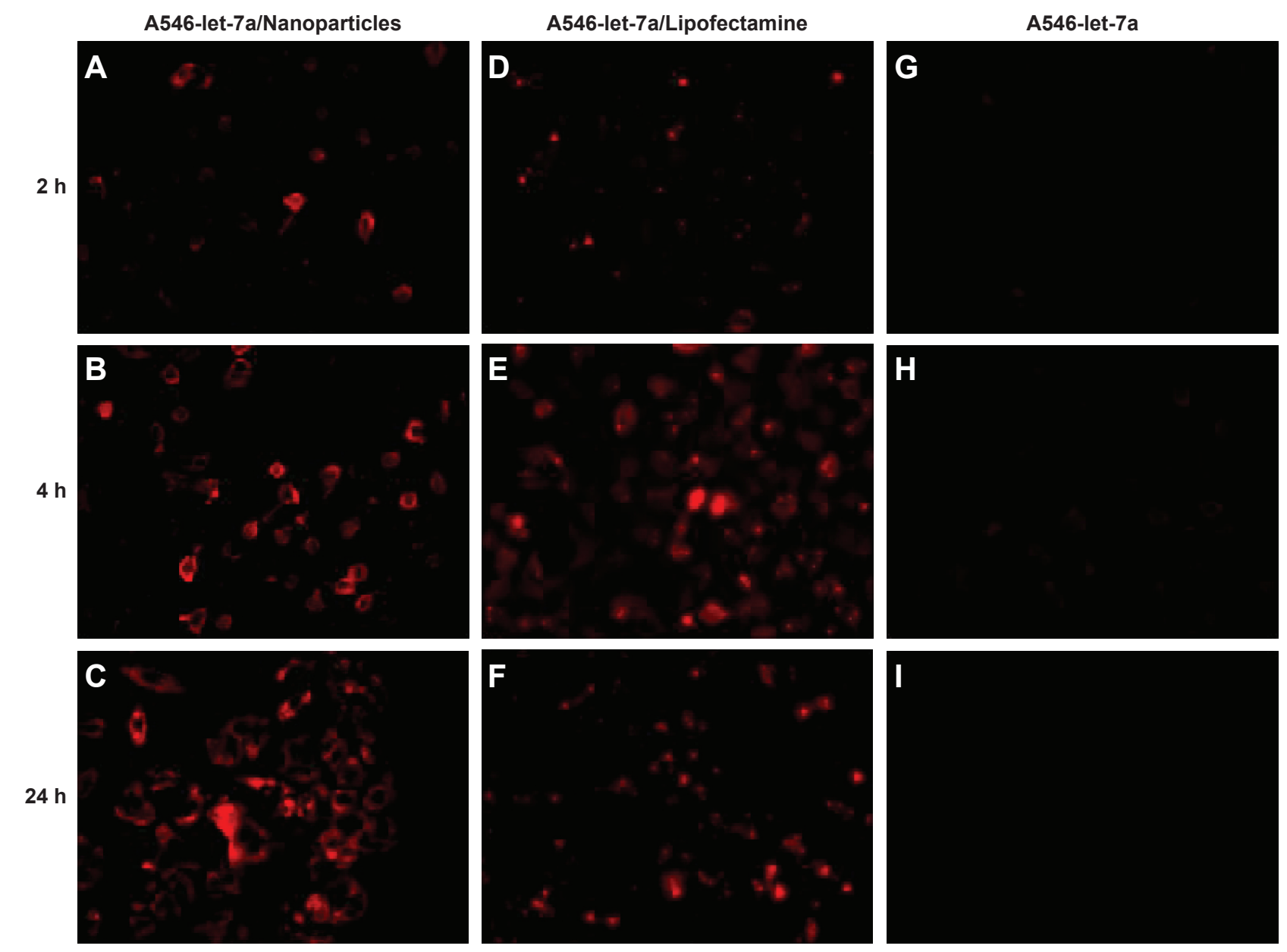

Figure SI Cellular uptake of fluorescently labeled A546-let-7a encapsulated with dextran nanoparticles. U-2OS cells were transfected with $100 \mathrm{nM}$ A546-let-7a (red) encapsulated with (A-C) dextran nanoparticles, (D-F) 100 nM A546-let-7a mixed with Life Technologies Lipofectamine ${ }^{\circledast}$ RNAiMAX (Thermo Fisher Scientific, Waltham, MA, USA), or (G-I) $100 \mathrm{nM}$ A546-let-7a alone. The cells were washed with phosphate-buffered saline and visualized by fluorescence microscopy at 2, 4, and 24 hours after transfection.

miR

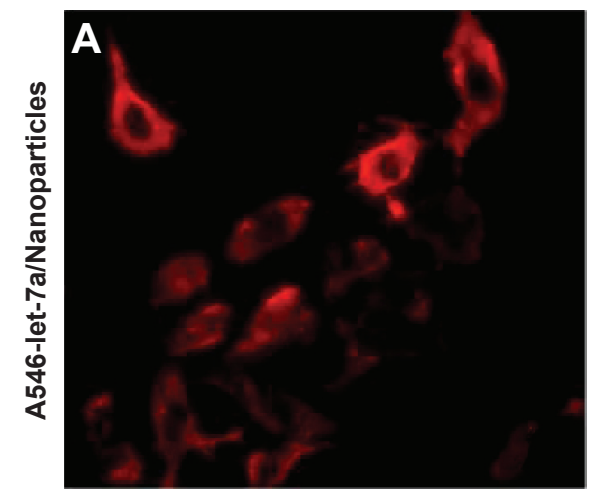

Nucleus

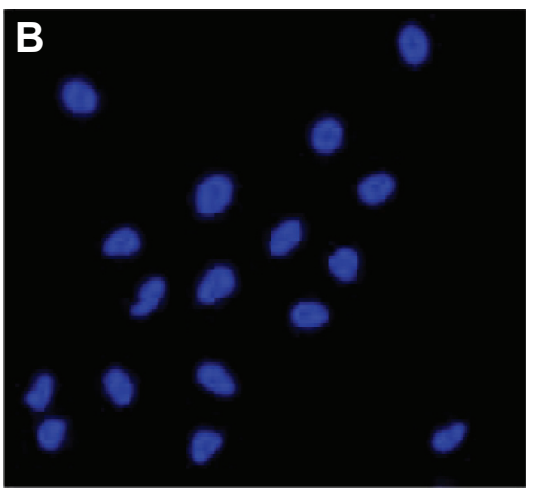

Merge

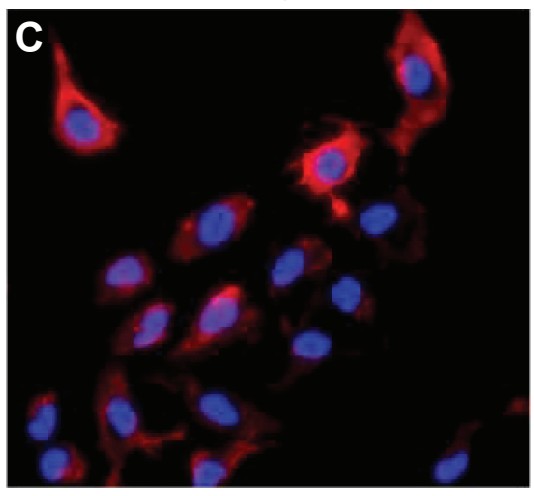

Figure S2 (Continued) 

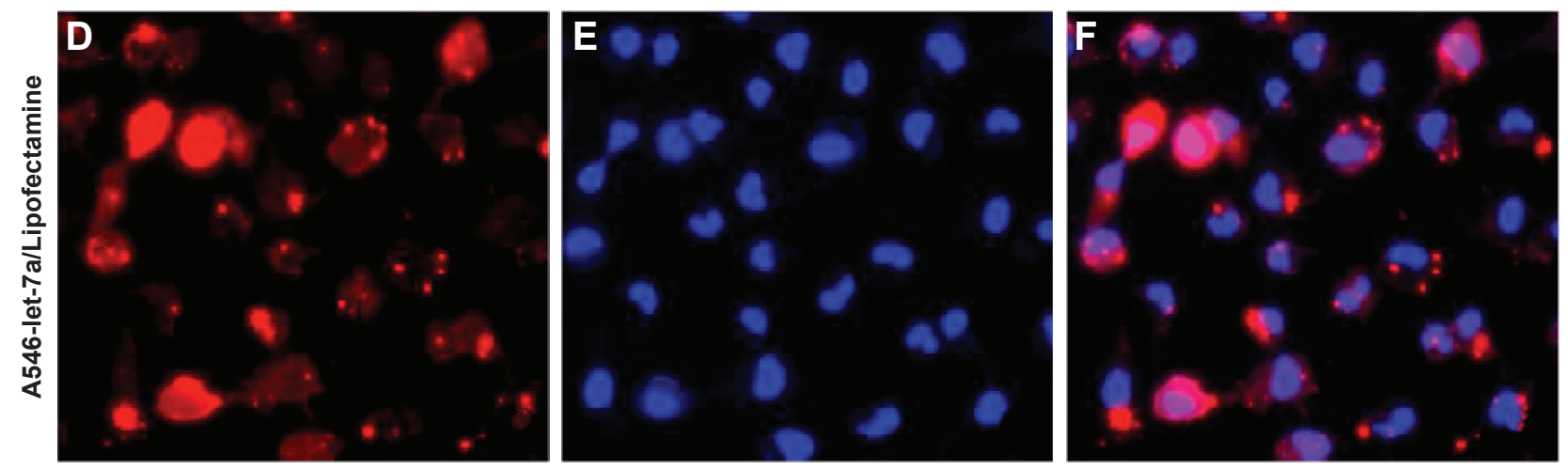

Figure S2 Subcellular distributions of fluorescently labeled A546-let-7a. U-2OS cells were transfected with (A-C) dextran-based nanoparticles or (D-F) Life Technologies Lipofectamine ${ }^{\circledast}$ RNAiMAX (Thermo Fisher Scientific, Waltham, MA, USA). Twenty-four hours after transfection, the cells were incubated with I $\mu \mathrm{g} / \mathrm{mL}$ Hoechst 33342 for I minute for nuclear counterstaining. Then, the cells were washed with phosphate-buffered saline and visualized under fluorescence microscope.

Abbreviation: miR, microRNA.

\section{Publish your work in this journal}

The International Journal of Nanomedicine is an international, peerreviewed journal focusing on the application of nanotechnology in diagnostics, therapeutics, and drug delivery systems throughout the biomedical field. This journal is indexed on PubMed Central, MedLine, CAS, SciSearch $\AA$, Current Contents ${ }^{\circledR} /$ Clinical Medicine,
Journal Citation Reports/Science Edition, EMBase, Scopus and the Elsevier Bibliographic databases. The manuscript management system is completely online and includes a very quick and fair peer-review system, which is all easy to use. Visit http://www.dovepress.com/ testimonials.php to read real quotes from published authors. 